\title{
Contenido de metales en muestras biológicas de la isla Rocuant, bahía Concepción, Chile
}

\section{Metal contents in biological samples of Rocuant island, Concepcion bay, Chile}

\author{
Camilo Werlinger I. ${ }^{1, *} \&$ Marco Salamanca $0 .{ }^{1}$ \\ 1Departamento de Oceanografía, Facultad de Ciencias Naturales y Oceanográficas, Universidad de Concepción, Concepción, \\ Chile. \\ *E-mail: cwerling@udec.cl
}

\begin{abstract}
RESUMEN
Se analiza la presencia de metales pesados en tejidos de organismos provenientes del sector aledaño a Isla Rocuant, Bahía Concepción. Los organismos estudiados fueron los bivalvos bentónicos Tagelus dombeii ("navajuela"), Mulinia edulis ("taquilla"), las macroalgas Gracilaria chilensis ("pelillo") y Rhodymenia sp ("rodymenia") y el pez Stromateus stellatus ("pampanito"). Los metales analizados en sus tejidos fueron $\mathrm{Cd}, \mathrm{Fe}, \mathrm{Hg}, \mathrm{Pb}$ y $\mathrm{Zn}$ para los cuales no existen registros previos en la localidad. La obtención de las muestras fue realizada por medio de buceo "hooka" para los organismos bentónicos, mientras que los peces fueron capturados mediante redes de pesca. En general, los metales encontrados presentan concentraciones comparables con los valores reportados en la literatura para las misma especies, pero en otras localidades, por lo que reflejan una condición natural, indicando además que los niveles de $\mathrm{Cd}, \mathrm{Hg}$ y $\mathrm{Pb}$ en los moluscos bivalvos se encuentran bajo los niveles de aceptación para productos de exportación. Se destaca además que en el caso del $\mathrm{Pb}$, este se encuentra bajo los niveles de detección analíticas en todos los organismos, al igual que el Cd en el pez $S$. stellatus y el alga G. chilensis. Otra diferencia que resalta corresponde a la presencia de Fe en Rhodymenia sp. que presenta el mayor valor absoluto de todo el estudio.
\end{abstract}

Palabras claVe: bentos, macroalgas, metales pesados, organismos marinos, peces.

\begin{abstract}
The presence of heavy metals in organisms tissues from the Rocuant Island, Concepcion Bay, is analyzed. The study was carried out on the bivalve Tagelus dombeii ("navajuela"), Mulinia edulis ("taquilla"), the seaweeds Gracilaria chilensis ("pelillo") and Rhodymenia sp ("rodymenia") and the fish Stromateus stellatus ("pampanito "). The metals analyzed were $\mathrm{Cd}, \mathrm{Fe}, \mathrm{Hg}, \mathrm{Pb}$ and $\mathrm{Zn}$ and there were no previous records in the locality. The sampling for benthic organisms was carried out by "hooka" diving, while fish were caught using a fishing nets. In general, metals concentrations are comparable to values reported in the literature for the same species in other areas of central Chilean coast. This suggests they reflect a natural condition and the levels of $\mathrm{Cd}, \mathrm{Hg}$ and $\mathrm{Pb}$ in the bivalve mollusks are under the acceptance levels for products to export. It should be noticed that $\mathrm{Pb}$ content in these organisms is under the analytical detection limits in all organisms, as well as $\mathrm{Cd}$ in the fish S. stellatus and the seaweeds G. chilensis. The Fe content in Rhodymenia sp. shows the highest absolute value of the whole study.
\end{abstract}

KEYwORDS: benthos, fishes, heavy metals, Rocuant Island, seaweeds.

\section{INTRODUCCIÓN}

El área costera de la bahía Concepción corresponde a una de las zonas de costa más intensamente utilizadas en el país y sometida a múltiples usos que involucran actividades industriales, turísticas, deportivas, navieras, portuarias, de recreación, ingeniería naval, etc; como también a actividades derivadas de una intensa intervención pesquera al encontrarse asociada a una de las áreas de extracción y captura de recursos más relevantes y productivas del país. Este uso múltiple ha significado un importante incremento de descargas de sustancias de diversa índole al ambiente marino entre los que se incluyen metales pesados y que ha derivado en varios conflictos entre diferentes usuarios siendo la contaminación de sus aguas y sedimentos un hecho reconocido y que ha alcanzado niveles críticos en algunos lugares bien localizados de la Bahía Concepción según lo han establecidos algunos estudios (Ahumada y 
Rudolph, 1987; Chuecas, 1989). La isla Rocuant o de los Reyes, ubicada al fondo de saco de la bahía ha sido una de las zonas que en la zona costera ha sufrido en forma intensa esta intervención siendo profundamente afectada por esos procesos de contaminación.

Dentro de los contaminantes inorgánicos la presencia de metales pesados en el ambiente marino puede provocar severos daños a la vida y a todo el ecosistema a consecuencia de sus efectos tóxicos que se ven potenciados por los fenómenos de acumulación en los seres vivos. Es así que elementos como $\mathrm{Hg}, \mathrm{Cd}, \mathrm{Pb}$, entre otros, son reconocidos por su toxicidad e impacto sobre la vida humana y los recursos costeros (Khan et al, 2015), lo que cobra un interés aún mayor como consecuencia de que estos metales, aún en concentraciones bajas, puede resultar altamente nocivos cuando son ingeridos durante largos periodos de tiempo.

En el presente estudio se entregan los resultados de análisis de metales pesados ( $\mathrm{Cd}, \mathrm{Fe}, \mathrm{Hg}, \mathrm{Pb}$ y $\mathrm{Zn}$ ) en muestras de peces, moluscos y macroalgas obtenidos en la
Isla Rocuant, Bahía Concepción, lugar del que se dispone escasa información sobre la presencia de estos elementos, y por consiguiente el objetivo de este estudio es el de aportar al conocimiento respecto de la presencia de estos elementos en organismos que se encuentran asociados a una zona que históricamente ha sufrido los fenómenos de contaminación anteriormente señalados.

\section{MATERIALES Y MÉTODOS}

\section{Muestreo}

Las muestras fueron obtenidas el 14 y 16 de Septiembre de 2015, en la Isla Rocuant, Bahía Concepción en los puntos que se indican en la Tabla1 y Figura 1. En el caso de los organismos bentónicos la obtención de los ejemplares se efectúo mediante buceo en las especies Tagelus dombeii (Lamarck, 1818) ("navajuela"), Mulinia edulis (King, 1832)(“taca"), Gracilaria chilensis C.J.Bird, McLachlan \& E.C.Oliveira, 1986 ("pelillo") y Rhodymenia sp. (Greville,

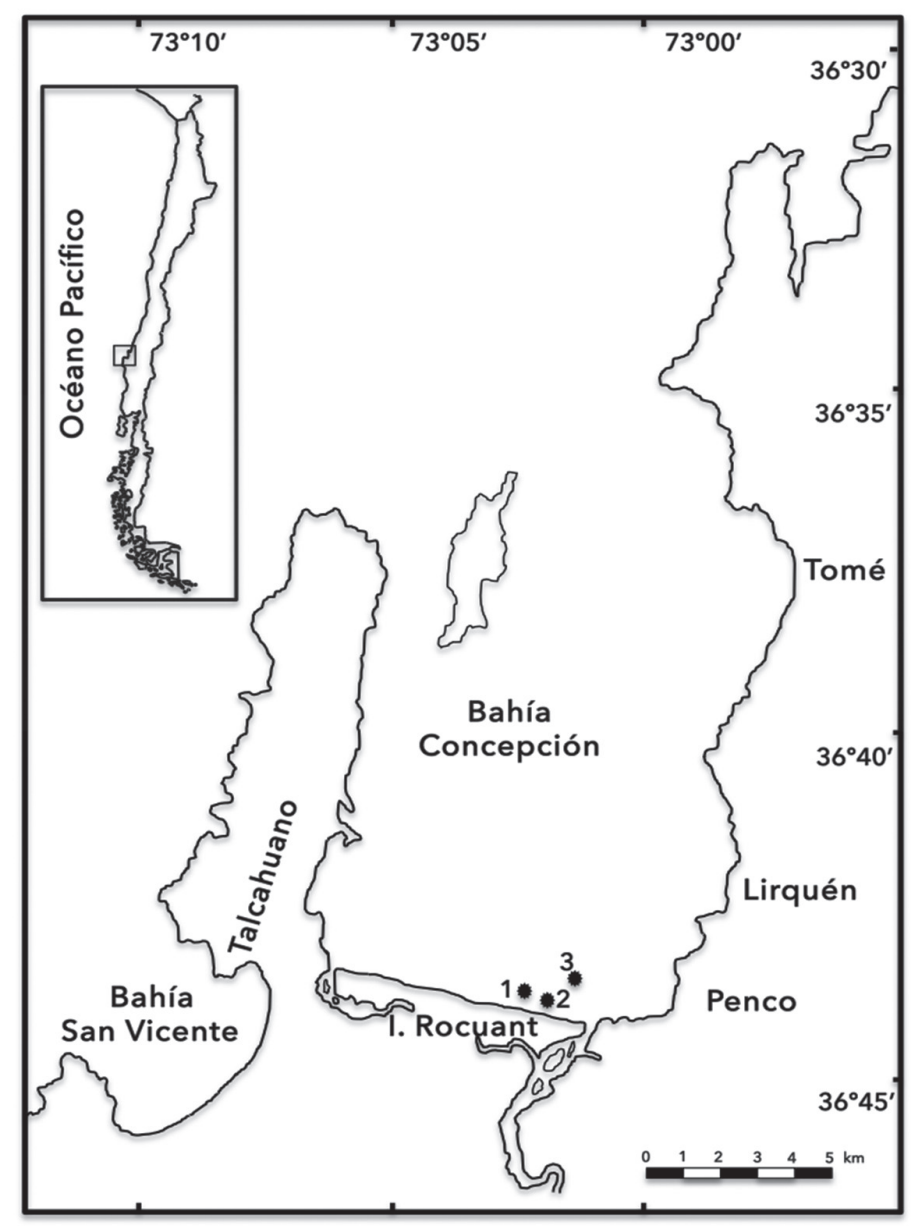

Figura 1. Mapa de Bahía de Concepción mostrando la ubicación de las áreas de muestreo. (1.- Tagelus dombeii, Mulinia edulis, Rodhymenia sp.; 2.- Gracilaria chilenis; 3.- Stromateus stellatus). / Concepcion Bay map showing the location of the sampling areas. (1.- Tagelus dombeii, Mulinia edulis, Rodhymenia sp.; 2.- Gracilaria chilenis; 3.- Stromateus stellatus). 
TABLA 1. Ubicación geográfica de los puntos de muestreo para las diferentes especies estudiadas. / Geographic location of sampling points for different species in the study.

\begin{tabular}{cccc}
\hline Punto Muestreo & Lat S & Long O & Organismos \\
\hline 1 & $36^{\circ} 43^{\prime}$ '54.02"S & $73^{\circ}$ 3'14.77'O & $\begin{array}{c}\text { Rhodymenia sp. } \\
\text { T. Dombey } \\
\text { M. Edulis }\end{array}$ \\
2 & & & G. Chilensis \\
3 & $36^{\circ} 44^{\prime} 4.96$ 'S & $73^{\circ} 1$ '27.89'O & S. stellatus \\
\hline
\end{tabular}

1830) ("Rodymenia") y en el caso de los peces mediante una red agallera capturando ejemplares de Stromateus stellatus Cuvier, 1833 ("pampanito"). En cada punto de muestreo se procedió a recoger un número variable de ejemplares de cada grupo de organismos en una cantidad suficiente para realizar los análisis químicos (aproximadamente 120 y 300 $\mathrm{g}$ de peso húmedo).

\section{PREPARACIÓN DE LAS MUESTRAS}

Los tejidos analizados realizados para cada organismo de los diferentes grupos fueron realizados en triplicado, los que se seleccionaron con un criterio de consumo humano, es decir en el caso del "pampanito" (Stromateus stellatus) se analizó tejido muscular, en la "navajuela" (Tagelus dombeii) el pie y en la "taca" (Mulinia edulis) el organismos entero. En las algas "pelillo" (Graciliaria chilensis) y "rodymenia" (Rhodymenia $s p$ ) se analizaron las frondas. Las muestra después de recolectada se guardaron en bolsas plástica selladas y se mantuvieron refrigeradas en un contendor de plumavit con material refrigerante hasta su traslado a laboratorio. Previo al análisis, las muestras se lavaron con agua desionizada y enjuagadas con agua Mili Q. Posteriormente los tejidos seleccionados fueron secados mediante liofilización, de tal forma que los resultados se expresan sobre base seca. Posteriormente los tejidos seleccionados fueron sometidos a una digestión acida en frío con una mezcla de $\mathrm{HCl}$ y $\mathrm{HNO}_{3}$ en una proporción de 3:1 y luego a una digestión en un sistema de microondas marca Milestone modelo ETHOS-D. acuerdo al método EPA 3052 para Cd, Fe, Pb y Zn. La muestra para la determinación de $\mathrm{Hg}$ se introduce directamente al mercuriometro (Método EPA 7473).

\section{Determinacion De Metales}

La solución resultante del ataque acido fueleída en un Espectrómetro de Absorción Atómica de Llama marca Thermo, Modelo Solaar 5, para la cuantificación de Cd, $\mathrm{Fe}, \mathrm{Pb}$ y $\mathrm{Zn}$. El $\mathrm{Hg}$ fue leído en un analizador directo de mercurio marca Milestone Modelo DMA-80 todos en el
Laboratorio de Oceanografía Química de la Universidad de Concepción.

\section{Aseguramiento de la Calidad Analítica}

Para el aseguramiento de la calidad analítica y control analítico ("QA/QC") de las muestras se utilizó un procedimiento que consintió en el uso de Materiales de Referencia Certificados (MRC) DORM 4 y TORT 2 del National Research Council de Canadá. Con esto se determinó la imprecisión e inexactitud como la desviación estándar de tres replicas y la desviación de mediciones respecto al valor informado en el certificado del MRC, respectivamente. Adicionalmente, en cada corrida analítica se leyeron blancos de reactivos y agua Mili Q al inicio y término de las lecturas instrumentales.

\section{RESULTADOS}

En la Tabla 2 se presenta el contenido promedio y la desviación estándar de los metales considerados por grupo de organismos. En esta tabla se observa que el $\mathrm{Cd}$ no fue detectado $\left(<0,1 \mu \mathrm{g} \mathrm{g}^{-1}\right)$ en el "pampanito" (Stromateus stellatus), la "navajuela" (Tagelus dombeii) y "pelillo" (Graciliaria chilensis). El contenido de $\mathrm{Pb}$ en todos los organismos analizados se encuentra bajo el límite de detección analítico $\left(<0,07 \mu \mathrm{g} \mathrm{g}^{-1}\right)$. El mayor contenido de Fe se presenta en el alga Rhodymenia sp. (i.e., 5908,0 $\pm 691,6$ $\mu \mathrm{g} \mathrm{g}^{-1}$ ) y el menor en Stromateus stallatus (i.e., $6,4 \pm 0,5 \mu \mathrm{g}$ $\left.\mathrm{g}^{-1}\right)$. El Hg muestra un patrón exactamente inverso, es decir el mayor contenido de este elemento se da en el "pampanito" (i.e., $0,164 \pm 0,0143 \mu \mathrm{g} \mathrm{g}^{-1}$ ) y el menor en el alga "rodymenia" (i.e., 0,024 $\pm 0,003 \mu \mathrm{g} \mathrm{g}^{-1}$ ). Finalmente el $\mathrm{Zn}$ muestra valores comparables entre los diferentes organismos, no obstante el contenido menor (i.e., 11,98 $\pm 0,29 \mu \mathrm{g} \mathrm{g}^{-1}$ ) se da en el "pampanito" cuya concentración es 4,7 veces menor al mayor contenido de $\mathrm{Zn}$ que se presenta en la "navajuela" (i.e., $56,28 \pm 0,65 \mu \mathrm{g} \mathrm{g}^{-1}$ ). 
TABLA 2. Contenido de metales en peces, bivalvos y macroalgas recolectados en Bahía Concepción. / Metals contents in fish, bivalves and macroalgae collected in Concepcion Bay.

\begin{tabular}{|c|c|c|c|c|c|c|c|c|c|c|}
\hline \multirow{2}{*}{$\begin{array}{c}\text { Metal } \\
\text { Organismo }\end{array}$} & \multicolumn{2}{|r|}{$C d$} & \multicolumn{2}{|r|}{$\mathrm{Fe}$} & \multicolumn{2}{|r|}{$H g$} & \multicolumn{2}{|r|}{$\mathrm{Pb}$} & \multicolumn{2}{|r|}{$Z n$} \\
\hline & $\begin{array}{c}\text { Promedio } \\
\left(\mu g g^{-1}\right)\end{array}$ & D.S. & $\begin{array}{c}\text { Promedio } \\
\left(\mu g g^{-1}\right)\end{array}$ & D.S. & $\begin{array}{c}\text { Promedio } \\
\left(\mu g g^{-1}\right)\end{array}$ & D.S. & $\begin{array}{c}\text { Promedio } \\
\left(\mu g g^{-1}\right)\end{array}$ & $D . S$. & $\begin{array}{c}\text { Promedio } \\
\left(\mu g g^{-1}\right)\end{array}$ & D.S. \\
\hline Stromateus stellatus & $<0,10$ & $<0,10$ & 6,4 & 0,5 & 0,162 & 0,0143 & $<0,07$ & $<0,07$ & 11,98 & 0,29 \\
\hline Tagelus dombeii & 0,62 & 0,03 & 177,2 & 7,3 & 0,053 & 0,0012 & $<0,07$ & $<0,07$ & 56,28 & 0,65 \\
\hline Mulinia edulis & $<0,10$ & $<0,10$ & 117,1 & 11,7 & 0,038 & 0,001 & $<0,07$ & $<0,07$ & 54,27 & 0,72 \\
\hline Gracilaria chilensis & $<0,10$ & $<0,10$ & 959,6 & 33,8 & 0,005 & 0,0004 & $<0,07$ & $<0,07$ & 32,65 & 0,54 \\
\hline Rhodymenia sp. & 0,13 & 0,02 & 5908,0 & 691,6 & 0,024 & 0,0003 & $<0,07$ & $<0,07$ & 48,84 & 0,61 \\
\hline
\end{tabular}

D.S.: Desviación Estándar

TABLA 3. Comparación del contenido de metales en los organismos y algas analizados en el presente estudio respecto de información recopilada en literatura. / Comparison of the metals content in organisms and algae analyzed in this study with respect to information from literature.

\begin{tabular}{|c|c|c|c|c|c|c|c|c|}
\hline Grupo & Especie & Localidad & Unidad & $\mathrm{Cd}$ & $\mathrm{Fe}$ & $\mathrm{Hg}$ & $\mathrm{Pb}$ & $\mathrm{Zn}$ \\
\hline \multirow[t]{2}{*}{ Peces } & \multirow[t]{2}{*}{ S. stellatus } & Desembocadura rio $\operatorname{Itata}^{(1)}$ & $\mu \mathrm{g} \mathrm{g}^{-1}$ & $0,65 \pm 0,51$ & $417 \pm 949$ & $0,09 \pm 0,03$ & $0,18 \pm 0,14$ & $40,4 \pm 22,9$ \\
\hline & & Este estudio & $\mu \mathrm{g} \mathrm{g}^{-1}$ & $<0,1$ & $6,4 \pm 0,5$ & $0,162 \pm 0,0143$ & $<0,07$ & $11,98 \pm 0,29$ \\
\hline \multirow{5}{*}{ Bivalvos } & \multirow{3}{*}{ T. dombeii } & Antofagasta $^{(2)}$ & $\mu \mathrm{g} \mathrm{g}^{-1}$ & 0,04 & - & - & 94 & 21 \\
\hline & & Bahia San Vicente ${ }^{(5)}$ & $\mu \mathrm{g} \mathrm{g}^{-1}$ & $3,53 \pm 0,31$ & - & $0,5 \pm 0,17$ & $0,53 \pm 0,4$ & $196,67 \pm 15,28$ \\
\hline & & Este estudio & $\mu \mathrm{g} \mathrm{g}^{-1}$ & $0,62 \pm 0,03$ & $177,2 \pm 7,3$ & $0,053 \pm 0,001$ & $<0,07$ & $56,3 \pm 0,7$ \\
\hline & \multirow[t]{2}{*}{ M. edulis } & Desembocadura rio $\operatorname{Itata}^{(1)}$ & $\mu \mathrm{g} \mathrm{g}^{-1}$ & 2,91 & 2402 & 0,04 & 0 & 94,9 \\
\hline & & Este estudio & $\mu \mathrm{g} \mathrm{g}^{-1}$ & $<0,1$ & $117,1 \pm 11,7$ & $0,038 \pm 0,001$ & $<0,07$ & $48,8 \pm 0,6$ \\
\hline \multirow{6}{*}{ Algas } & M. pirifera & Lirquen, Bahía Concepción ${ }^{(3)}$ & $\mu \mathrm{g} \mathrm{g}^{-1}$ & $3,7 \pm 1,5$ & - & - & - & - \\
\hline & I. laminaroides & Bahia San Vicente ${ }^{(4)}$ & $\mu \mathrm{g} \mathrm{g}^{-1}$ & $1,7 \pm 0,9$ & - & $0,21 \pm 0,07$ & $9,86 \pm 3,93$ & $237 \pm 54,3$ \\
\hline & I. laminaroides & Bahia Coliumo $^{(4)}$ & $\mu \mathrm{g} \mathrm{g}^{-1}$ & $4,1 \pm 0,2$ & - & $0,09 \pm 0,07$ & $1,08 \pm 0,6$ & $17,6 \pm 6,96$ \\
\hline & I. laminaroides & Quidico $^{(4)}$ & $\mu \mathrm{g} \mathrm{g}^{-1}$ & $5,6 \pm 0,6$ & - & $0,04 \pm 0,04$ & $1,44 \pm 1,7$ & $14,9 \pm 3,45$ \\
\hline & G. chilensis & Este estudio & $\mu g \mathrm{~g}^{-1}$ & $<0,1$ & $969 \pm 34$ & $0,005 \pm 0,0004$ & $<0,07$ & $32,7 \pm 0,5$ \\
\hline & Rhodymenia.sp. & Este estudio & $\mu \mathrm{g} \mathrm{g}^{-1}$ & 0,13 & $5908 \pm 691$ & $0,024 \pm 0,0003$ & $<0,07$ & $48,8 \pm 0,6$ \\
\hline
\end{tabular}

(1): PROMNA, 2014

(2): Castro y Valdez, 2012

(3): Beltran et al, 1993

(4): Encina, 1993

(5): Ahumada, 1994

\section{DISCUSIÓN}

En la Tabla 3 se presenta, con fines referenciales, una recopilación del contenido de los metales estudiados en el presente estudio con resultados obtenidos en la zona costera de la desembocadura del río Itata, al norte de Bahía Concepción, que representa la mejor base de datos ambientales marinos con que se cuenta hoy en día en la VIII región, obtenidos por el Programa de Monitoreo Marino (PROMNA) del
CFI Nueva Aldea (Universidad de Concepción, 2014). La información recolectada en este programa es sobre de Stromateus stellatus y Mulinia edulis. En Tagelus dombeii se obtuvo información en Bahía San Jorge en Antofagasta para $\mathrm{Cd}, \mathrm{Pb}$ y $\mathrm{Zn}$ y en Bahía San Vicente para $\mathrm{Cd}, \mathrm{Hg}, \mathrm{Pb}$ y $\mathrm{Zn}$. Se debe mencionar que en general la información del contenido de metales en algas es mucho más limitada con respecto a otros grupos de organismos, y no se encontró información para las dos algas consideradas en el presente 
estudio, sin embargo en la Tabla 3 se ha incluido, con fines referenciales contenido de metales en otras algas (i.e., Iiridea laminaroides y Macrocytis pirifera).

En general, los contenidos de metales obtenidos en el presente estudio son comparables con los valores reportados en literatura para otras localidades costeras de Chile. Esto quiere decir que los niveles de metales encontrados en los organismos evaluados están en el rango de concentración informados para el mismo pez y moluscos bivalvos en otras áreas lejanas geográficamente, por lo que reflejan una condición natural. Adicionalmente, los niveles de $\mathrm{Cd}, \mathrm{Hg}$ y $\mathrm{Pb}$ informados para los moluscos bivalvos evaluados en este estudio están bajo el límite de aceptación (1,0, 0,5 y 1,5 $\mu \mathrm{g}$ $\mathrm{g}^{-1}$, respectivamente) considerado en el Programa de Sanidad de Moluscos Bivalvos (PSMB) del Servicio Nacional de Pesca para la exportación de moluscos bivalvos a la Unión Europea, Estados Unidos y Singapur (fide INTIMIT, 2013). Las diferencias con este patrón están dadas por la detección bajo el límite de detección analítico para el caso de $\mathrm{Pb}$ en todos los organismos, y para $\mathrm{Cd}$ en el pez $S$. stellatus y el alga $G$. chilensis. La otra diferencia está dada por la concentración de Fe en Rhodymenia sp. que presenta el valor absoluto mayor de todo el estudio; e igualmente, pero en sentido inverso en Tagelus dombeii, en donde los valores reportados en el estudio de Ahumada (1994) para Cd, $\mathrm{Hg}$, $\mathrm{Pb}$ y $\mathrm{Zn}$, son considerablemente mayores alcanzando concentraciones de entre 3,5 a 9,5 veces por sobre las concentraciones detectadas en este estudio.

\section{AGRADECIMIENTOS}

Los autores agradecen a GNL Talcahuano por permitir la publicación de estos datos que corresponden a información obtenida a partir de un estudio de línea base encargado al Departamento de Oceanografía de la Universidad de Concepción. Igualmente agradecemos al Sr. Braulio Fernández Z., Alumno de la Carrera de Biología Marina de la Universidad de Concepción por su valiosa ayuda en la toma de muestras en terreno, al igual que a los alumnos tesistas de la misma carrera Sres. Marco Hidalgo G. y Luis Bermedo C., y el buzo Sr. Ignacio Rudolph K. que apoyaron la realización del muestreo.

\section{REFERENCIAS}

Ahumada, R., Rudolph, A. 1987. Un problema crítico de contaminación por orgánicos. Marisma Rocuant en Talcahuano. Revista Ciencia y Tecnología del Mar. CONA 11: 45-59.

Ahumada, R. 1994. Nivel de concentración e índice de bioacumulación para metales pesados $(\mathrm{Cd}, \mathrm{Cr}, \mathrm{Cu}, \mathrm{Hg}$, $\mathrm{Ni}, \mathrm{Pb}$ and $\mathrm{Zn}$ ) en tejido de invertebrados bénticos de bahía San Vicente, Chile. Revista de Biología Marina y Oceanografía 29(1): 77-87.

Beltrán, C., Manríquez, E., Soto, M., Fernández, E. 1993. Determinación de Cadmio en Macrocystis pyrifera, Cancer coronatus y Aulacomya ater del Sector Lirquén, Bahía Concepción, Chile. Libro Resúmenes XIII Jornadas Ciencias del Mar. Viña del Mar. Pág. 51

Castro, G., Valdés, J. 2012. Concentración de metales pesados $(\mathrm{Cu}, \mathrm{Ni}, \mathrm{Zn}, \mathrm{Cd}, \mathrm{Pb})$ en la biota y sedimentos de una playa artificial, en la bahía San Jorge $23^{\circ} \mathrm{S}$, norte de Chile. Latin American Journal of Aquatic Research 40(2): 267-281.

Chuecas, L. 1989. Contaminación por metales pesados en el litoral de la Región del Bío-Bío, Concepción, Chile: el caso del mercurio y el cadmio. Ambiente y Desarrollo 5(1): 137145.

EncinA, F. 1993. Estudio de metales (Cu, Zn, Pb, $\mathrm{Cd}$ y Hg) en Iridaea laminarioides, Iridaea ciliata y Gracilaria chilensis (Rhodophyta: Gigartinales) en las Bahías San Vicente, Coliumo y Quidico (Octava Región, Chile). Tesis para optar al Grado de Doctor en Ciencias Ambientales. Universidad de Concepción. 60 pp.

Instituto Tecnológico De Mitilicultura. 2013. Evaluación comparativa de variables que influyen en la engorda del mejillón chileno a través de un Sistema de Información Geográfico. (SIG, basado en el Programa Sanitario de Moluscos Bivalvos). Informe Final Proyecto Colaboración EuroChile-Fundación Empresarial Comunidad Europea, Chile. 31 pp.

Khan, N., Ryu, K.Y., Choi, J.Y., Nho, E.Y., Habte, G., Choi, H., KIM, M.H., PARK, K.S., KIM, K.S. 2015. Determination of toxic heavy metals and speciation of arsenic in seaweeds from South Korea. Food Chemistry169: 464-70.

Universidad De Concepción. 2014. Informe Programa de Monitoreo del Medio Marino. Campañas 1-33. CFI Nueva Aldea. Fotocopiado. 600 pp. 